\title{
TUBERCULOUS MENINGITIS IN TWO BROTHERS VACCINATED WITH B.C.G.*
}

\author{
BY \\ R. MCLAREN TODD \\ From the Department of Child Health, University of Liverpool
}

(RECEIVED FOR PUBLICATION NOVEMBER 1, 1954)

Bacille-Calmette-Guérin (B.C.G.) vaccination on a large scale was started in a number of European countries in 1947 by the Danish Red Cross, and in the following year this campaign was enlarged under the United Nations International Children's Emergency Fund (Unicef) and became known as the 'Joint Enterprise'. As a result of this scheme, some 8 million children and young adults have been vaccinated with B.C.G. Bearing in mind the Lübeck disaster of 1930 when 73 vaccinated children died from tuberculosis, it is especially important when any large-scale vaccination project is undertaken to be satisfied that the technical arrangements are such that severe complications are unlikely to develop. If complications are encountered the circumstances should be fully investigated because complications might have serious repercussions on future B.C.G. vaccination campaigns, and also on other forms of prophylaxis, such as diphtheria immunization, which have been successful in reducing mortality and morbidity. Up to the present time few serious complications have been reported in children following B.C.G. vaccination, but it is the purpose of this communication to record the development of tuberculous meningitis in two brothers vaccinated with B.C.G., to review the relevant literature, and to discuss the implications.

\section{Case Reports}

Case 1. G.W., born on October 31, 1950, was vaccinated with B.C.G. $(0.1 \mathrm{ml}$. of batch 1006) when he was 21 months old. A Mantoux test had been performed 23 days before vaccination and the reaction was negative. His father was suffering from open pulmonary tuberculosis and had been removed to a sanatorium two months previously. Thirty-one days after vaccination the child wanted to be nursed continuously, was generally off colour and developed a cough. One week later he became anorexic and began to lose weight; after a further week he began to vomit, was constipated, developed

- A paper read at a joint meeting of the Birmingham, Leeds. Liverpool and Sheffield Paediatric Clubs at Sheffield on September 25, 1954. grunting respirations and had a temperature of $103^{\circ} \mathrm{F}$. He was given sulphonamides for four days but no improvement in his condition was apparent. On admission to hospital (52 days after B.C.G. vaccination) he had a temperature of $101^{\circ} \mathrm{F}$., pulse rate of 140 and respiration rate of 30 per minute. No definite abnormal signs were present in the lungs or elsewhere. A blood count showed Hb $90 \%$, white cell count 15,000 per c.mm. $(80 \%$ polymorphs) and the sedimentation rate was $12 \mathrm{~mm}$. in one hour. Lumbar puncture was performed and a clear, colourless cerebrospinal fluid, which developed a spiderweb clot on standing, was obtained. The fluid contained $100 \mathrm{mg}$. ${ }^{\circ}$ o protein, $32 \mathrm{mg}$. ${ }^{\circ}$ o sugar, 180 cells per c.mm. $\left(90^{\circ}\right.$ o mononuclears) but acid-fast bacilli were not seen on direct examination. Treatment with intramuscular and intrathecal streptomycin and oral I.N.H. was started and he was transferred to Alder Hey Children's Hospital.

On examination he was a pale, wasted, semicomatose child with marked neck stiffness and a positive Kernig's sign. He had a left facial palsy and spasticity of the right arm and right leg. A small scar was present over the left deltoid at the site of B.C.G. vaccination. The margins of the optic dises were blurred but no choroidal tubercles were seen. The Mantoux reaction was negative at 1 in 10,000 dilution but positive at 1 in 1,000 and a radiograph of the chest showed a primary complex at the right lung base. Lumbar puncture was performed and tubercle bacilli were grown from the cerebrospinal fluid; guinea-pigs inoculated with the fluid developed typical tuberculous lesions; the tubercle bacilli were of the human type and were sensitive to streptomycin and I.N.H. Treatment with streptomycin and I.N.H., which he was having on admission, was continued but his condition deteriorated, papilloedema and spreading of the skull sutures developed, and burr holes were made through which streptomycin was given into the lateral ventricles. His condition further deteriorated and intrathecal P.P.D. was given daily for eight weeks (Fig. 1). He then made slow but steady progress, and with the help of physiotherapy the right hemiplegia improved. The meningitis is now inactive and has remained so during the past 18 months; his vision is normal but he is deaf.

Case 2. R.W., born on December 21, 1946, was vaccinated with B.C.G. $(0 \cdot 1 \mathrm{ml}$. of batch 1006$)$ when he 


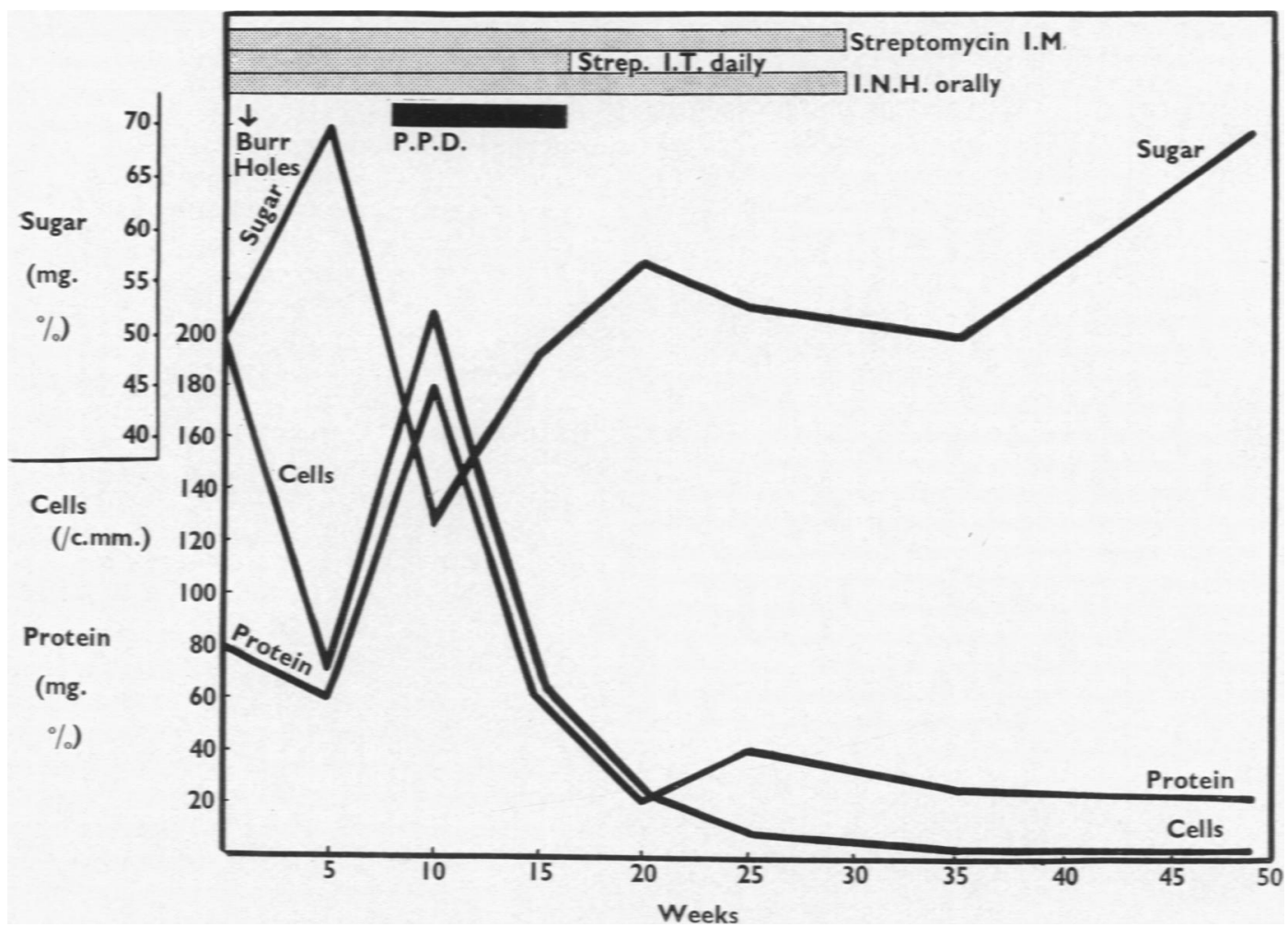

Fig. 1. Case 1.

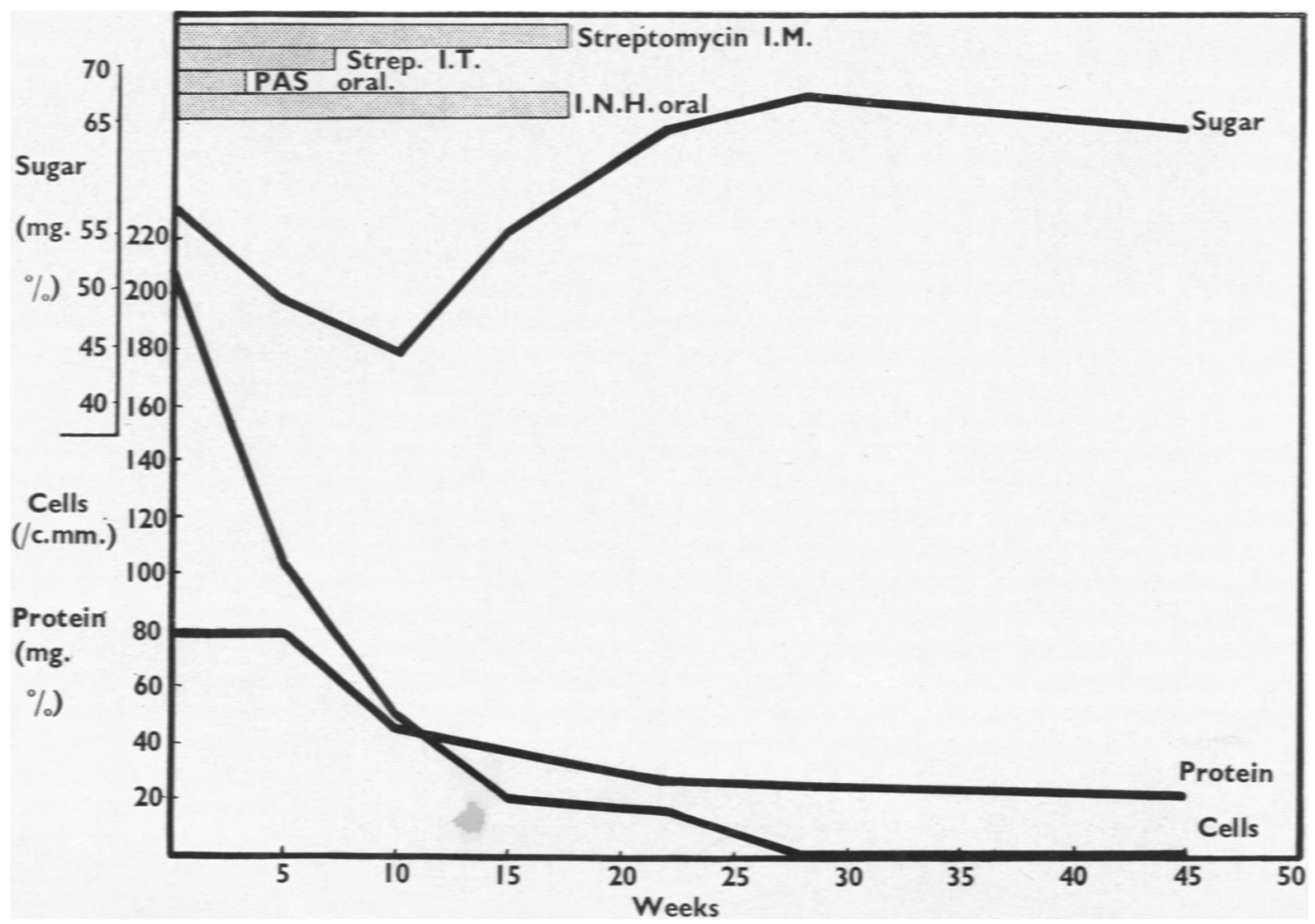

Fig. 2. Case 2. 
was $5 \frac{1}{2}$ years old, on the same day as his younger brother. A Mantoux reaction 23 days previously was negative, and when seen 54 days after vaccination the Mantoux reaction $(1$ in 1,000$)$ was positive (erythema $35 \mathrm{~mm}$. in diameter, induration $20 \mathrm{~mm}$. in diameter) and he had a vesicle $6 \mathrm{~mm}$. in diameter at the vaccination site. He had been in good health both before and after vaccination, and he remained well until six months later when, after returning from school, he complained of headache and vomited on one occasion. Two days later he was admitted to hospital with a temperature of $101^{\circ} \mathrm{F}$. He was fully conscious, the optic fundi were normal and the only abnormal finding was slight neck stiffness. A chest radiograph showed large glands at the right hilum and shadowing in the right upper lobe. Lumbar puncture revealed a clear, colourless cerebrospinal fluid which developed a spider-web clot on standing. The fluid contained 210 lymphocytes per c.mm., $80 \mathrm{mg}$. ${ }^{\circ}$ o protein, $58 \mathrm{mg} .{ }^{\circ}$ o sugar, and tubercle bacilli were seen on direct examination. The fluid was cultured but no growth obtained. The fluid was also inoculated into a guineapig but the animal died prematurely and showed no evidence of tuberculosis. He was treated with intramuscular streptomycin, intrathecal streptomycin and oral I.N.H. and made an uninterrupted recovery (Fig. 2).

His father, who was in a sanatorium at the time B.C.G. vaccination was performed, had returned home and was sputum-negative following a thoracoplasty, but further investigation of other members of the household previously free from tuberculosis showed that an aunt had radiological evidence of active tuberculosis. It is probable that the boy had been infected recently from this source.

\section{Discussion}

The development of tuberculous meningitis in relation to B.C.G. vaccination is a rare occurrence; indeed it is commonly assumed that tuberculous meningitis and miliary tuberculosis do not occur in B.C.G.-vaccinated children. There is indirect evidence that the frequency of these complications is much less in children who have received B.C.G. vaccination when compared with children not vaccinated, but there is no convincing statistical proof. In any large-scale vaccination project isolation of the children to be vaccinated for a preliminary six-week period and for a similar period after vaccination is impracticable. In these circumstances it is inevitable that some of the children will be already infected with tuberculosis at the time of vaccination, although still in the preallergic phase in which the Mantoux test is negative. Others may become infected with tuberculosis after vaccination but before this procedure could have any influence upon the development of a tuberculous lesion. The incubation period for tuberculosis is generally regarded as being from 35 to 40 days, but in some recorded instances the lower limit has been 15 days and the upper limit 56 days. If, therefore, a patient presents evidence of tuberculosis within 15 days of vaccination we can conclude that the disease was present before vaccination; if tuberculosis develops more than 56 days after vaccination it is probable that the disease was acquired after vaccination; if evidence of tuberculosis exists 15 to 56 days after vaccination we cannot be sure if it was acquired before or after vaccination. In any event, there is no evidence to suggest that B.C.G. vaccination shortly before or after infection with tuberculosis affects the course of the disease, so that the precise time of development of tuberculosis in relation to B.C.G. vaccination may be purely an academic point and of no practical significance. But the development of a serious form of tuberculosis in close relation to B.C.G. vaccination may influence the public in their acceptance of this form of prophylaxis.

Imerslund (1943) described the subsequent clinical findings in a boy of 13 years who was vaccinated with B.C.G. The Mantoux reaction was negative one week before vaccination and Mantoux conversion developed six weeks later. Eleven months after vaccination he developed tuberculous meningitis; human tubercle bacilli were grown from the cerebrospinal fluid and at necropsy the findings were characteristic of tuberculous meningitis. Rosenthal, Leslie and Loewinsohn (1948) vaccinated a newborn infant with B.C.G. A patch test at the age of 6 months and again at 18 months of age was weakly positive, but at 15 months it was negative. At 22 months of age the infant died from tuberculous meningitis. It is probable that in this patient B.C.G. produced little or no immunity to tuberculosis. Wasz-Höckert (1949) has published observations on twins who were given B.C.G. vaccine three days after birth. In both children Mantoux conversion took place, but in one of them tuberculous meningitis developed six and a half months after vaccination, the diagnosis being confirmed at necropsy. The other twin remained healthy. Savilathi's patient (quoted by Ustvedt, 1951) developed pleurisy six months after successful vaccination with B.C.G. and died from tuberculous meningitis two years later. Lichtenstein (quoted by Ustvedt, 1951) observed an infant who was vaccinated with B.C.G. at birth and in whom the Mantoux reaction became positive but tuberculous meningitis developed two and a half years later. In the Joint Enterprise (Ustvedt, 1951) tuberculous meningitis developed in 16 patients, and miliary tuberculosis in a further five patients, among the 8 million vaccinated. Unfortunately the clinical features of these patients have not been described in detail, but it is clear that at least 10 of the 16 patients 
developed tuberculous meningitis within eight weeks of B.C.G. vaccination. Dorothy Price (1954) analysed the results of 140,697 B.C.G. vaccinations performed throughout the Republic of Ireland. Tuberculous disease developed in 21 of these patients but in 11 of them tuberculosis was diagnosed within a few weeks of B.C.G. vaccination and in three patients the diagnosis was not confirmed. One girl of 12 years developed tuberculous meningitis 11 months after successful vaccination and she recovered from the disease. A boy of 6 years became ill 14 months after successful vaccination and died from what was thought to be tuberculous meningitis, but this diagnosis was based on clinical opinion and was not supported by radiological or bacteriological evidence.

The first of the two patients I have described developed tuberculous meningitis seven weeks after B.C.G. vaccination and it seems reasonably certain that he was in the pre-allergic phase of tuberculosis before vaccination was performed. The course of events in the second patient is more difficult to interpret. He was vaccinated on the same day as his brother and six weeks later Mantoux conversion had taken place. He was not in contact with a known case of open pulmonary tuberculosis during this period and he did not present any symptoms even of minor illness. The Mantoux test was performed with a tuberculin solution that was known to be potent, but a second test was not done before vaccination to be sure that he definitely had a negative tuberculin reaction. A radiograph of the chest was not performed at this stage to confirm the absence of pulmonary disease, but a radiograph taken six months later did show an active primary tuberculous complex. It is possible, though unlikely, that he contracted his primary infection from an unknown source at about the same time that B.C.G. vaccine was given, and what was interpreted as Mantoux conversion following B.C.G. was the result of a concurrent primary infection.

It is of interest to compare the response of these two brothers to treatment, contrasted in Figs. 1 and 2, although it could be argued that such a comparison is not valid because of the age difference and the different stage of the disease when treatment was begun. It did seem, however, that response to therapy in the second patient was more rapid than in other patients of the same age, treated at the same stage in their disease, and it is interesting to speculate upon the possible beneficial effects of B.C.G. vaccination on the course of tuberculous meningitis. The following table summarizes the response to treatment in these two patients:

\begin{tabular}{|c|c|c|}
\hline & Patient 1 & Patient 2 \\
\hline $\begin{array}{l}\text { Clinical response to } \\
\text { therapy }\end{array}$ & Slow & Quick \\
\hline Temperature & $\begin{array}{l}\text { Raised until 16th } \\
\text { week }\end{array}$ & $\begin{array}{c}\text { Normal after } 12 \text { th } \\
\text { day }\end{array}$ \\
\hline Appetite & Poor for 5 months & Normal throughout \\
\hline Weight & $\begin{array}{l}\text { No gain for first } \\
16 \text { weeks }\end{array}$ & $\begin{array}{l}\text { Steady gain from } 1 \mathrm{st} \\
\text { week }\end{array}$ \\
\hline $\begin{array}{l}\text { Duration of intrathecal } \\
\text { streptomycin }\end{array}$ & 17 weeks & 7 weeks \\
\hline $\begin{array}{l}\text { Duration of intramus- } \\
\text { cular streptomycin }\end{array}$ & 30 weeks & 17 weeks \\
\hline Duration of oral I.N.H. & 30 weeks & 17 weeks \\
\hline
\end{tabular}

\section{Summary}

The development of tuberculous meningitis in relation to B.C.G. vaccination is a rare event, and the chance of this complication arising, based upon the reported cases, is in the order of 1 in every 400,000 vaccinations. In at least half of these cases meningitis developed while the patients were in the pre-allergic stage of the disease and before vaccination could have any beneficial effect. In a survey of the literature 12 children were found who developed tuberculous meningitis several months after successful B.C.G. vaccination, and a further case is described in this paper. The response of this patient to the accepted treatment for tuberculous meningitis appeared to be more rapid than in patients not vaccinated with B.C.G.

REFERENCES

Imerslund. Olga (1943). Acta tuberc. scand., 17, 282.

Price, Dorothy S. (1954). Irish J. med. Sci., 6 ser., no. 338, p. 56. Rosenthal, S. R., Leslie, E. I. and Loewinsohn, E. (1948). J. Amer. med. Ass., 136, 73

Ustvedt, H. J. (1951). Conference on European B.C.G. Programmes. Copenhagen, 1949, ch. 13 . Heinemann, London.

Wasz-Höckert, O. (1949). Acta paediat.. L'ppsala, 37, 261 